\title{
Nubian ibex in south-western Jordan (Dana Nature Reserve)
}

\section{During a survey in Dana Nature Reserve (south- western Jordan) carried out between November 1994 and January 1995, Nubian ibexes Capra nubiana were sighted twice on a cliff at Jebel Um- Suwwana in the Wadi Araba area. The low number of sightings and an assessment of range conditions throughout the study area suggest that the population is reduced to a low density and suffers from severe competition for food and range with goats. Further research needs are discussed in a conservation perspective.}

The Nubian ibex Capra nubiana is currently distributed in the Middle East, including in Jordan, Palestine, Israel, Saudi Arabia and the United Arab Emirates (Harrison and Bates, 1991; Habibi, 1994). Few data are available on the status of the species, which is listed as Indeterminate by IUCN (Groombridge, 1993). Habibi (1994) reported the population to be reduced to small scattered nuclei throughout its range, being still widespread only in Israel. The dramatic reduction in range and numbers is believed to stem from a steady increase in human population, habitat destruction and deforestation, pasture deterioration by overgrazing from livestock and direct persecution (Habibi, 1994).

The only report on the Nubian ibex in Jordan is by Hatough-Bouran and Disi (1991), who described the species as occurring at low densities in a few scattered sites in western Jordan, including Wadi Mujib, Rum and Dana wildlife reserves. Its conservation status in Jordan has not yet been clearly established and there is no reliable information on demography and reproduction.

We conducted a survey to obtain information on the status and distribution of the Nubian ibex in Jordan's Dana Nature Reserve (DNR, Figure 1) within a broader United Nations Development Programme/World Bank Gobal Environmental Facility project for the conservation of Dana Wildlands, in collaboration with the Royal Society for the Conservation of Nature (RSCN) of Jordan (Catullo and Ciucci, 1995). The DNR (230 sq $\mathrm{km}$ ) is situated in south-western Jordan (Tafila district) and is characterized by a complex system of wadis and mountains with elevation

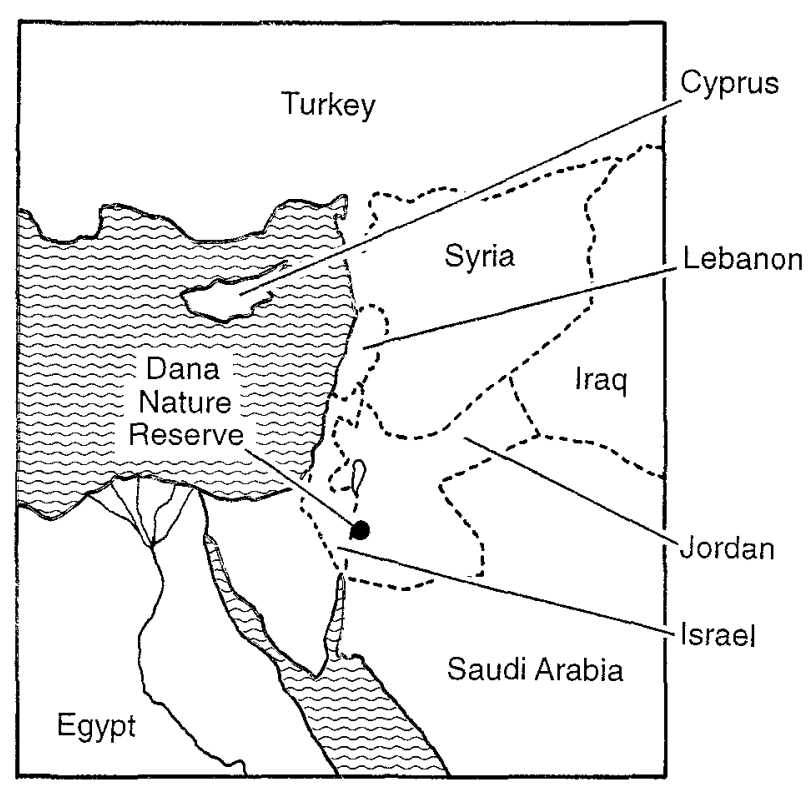

222
Figure 1. Location of Dana Nature Reserve.

(C) $1996 \mathrm{FFl}$, Oryx, 30 (3), 222-224 


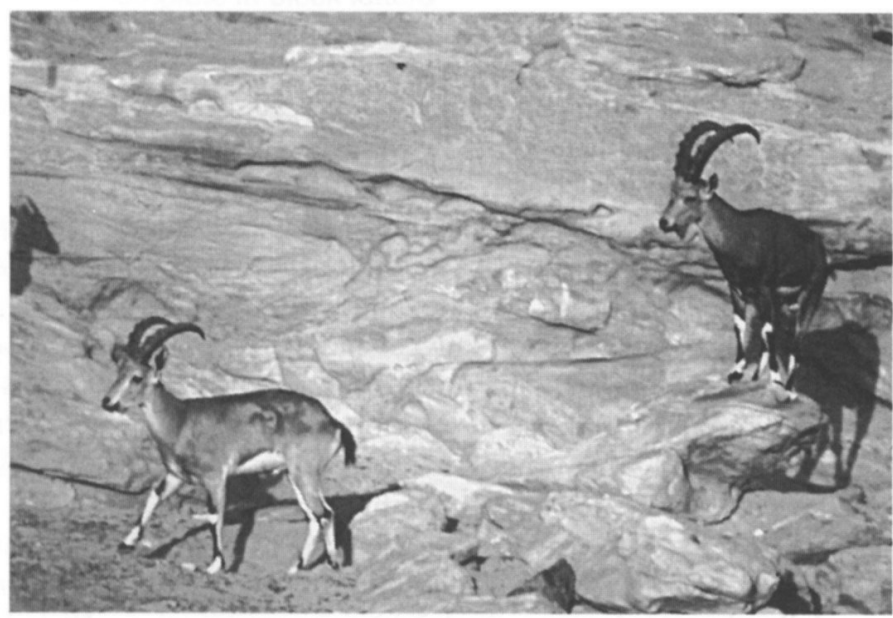

ranging from below sea level to $1500 \mathrm{~m}$. The reserve contains four major vegetation types (RSCN, 1994): sand-dune desert; acacia subtropical woodland; Irano-Turanian mid-altitude steppe; Mediterranean semiarid chaparral.

Between November 1994 and January 1995 data were gathered by direct observations, searches for ibex signs, range surveys and interviews with local residents. Observations were carried out using binoculars $(8 \times 30,10 \times$ $50)$ and telescopes $(20-45 \times)$ from selected vantage points, and sex/age categories were defined following Schaller (1977), Fox et al. (1992) and Habibi (1994). Thirteen vantage points were selected to account for a wide sampled area, good visibility of suitable ibex habitat, previously reported presence of ibexes, and proximity to water sources, pastures and escape terrains. The area sampled visually was $16.13 \mathrm{sq} \mathrm{km} \mathrm{(0.007} \mathrm{per} \mathrm{cent} \mathrm{of}$ DNR) and included 23 per cent of the estimated suitable ibex habitat. Availability of foraging areas was estimated visually according to the quality of soil and vegetation. Areas of critical habitat for ibex were located according to: (i) availability of escape terrains (Fox et al., 1992); (ii) proximity of escape terrains to foraging areas; (iii) availability of water sources; (iv) inaccessibility to humans.

A minimum estimate of ibex critical habitat is $70.1 \mathrm{sq} \mathrm{km}$ within the entire reserve, including the RSCN camp-site area, Khushom Ismail mountain area and Wadi el Mahash area. Observation effort totalled 97 hours, distributed at dawn ( 35 per cent) and sunset ( 44 per cent) with the rest scattered during the rest of the day (21 per cent). Ibexes were seen on only two occasions, for a total of 3.20 hours. Both observations involved only adult males and were in the same general location - the southfacing cliff of Jebel Um-Suwwana - within the critical habitat area of Wadi el Mahash. The limited number of sightings during the survey suggests a population at a critically low density, even though the small sample size does not provide meaningful indications about the demographic and reproductive status of the population. The preliminary results from this status survey strongly suggest that the local ibex population suffers from severe competition for food and range with livestock. Severe deterioration was detected in most of the available pasture and livestock (mostly goats) are widespread over the entire reserve; their impact, in terms of persistent and intensive grazing for most of the year, leads to soil erosion and desertification. Less than 4 per cent of the sampled pasture was of good quality, whereas about 16 per cent suffered from low to medium grazing pressure, and 80 per cent were characterized by intensive overgrazing. Livestock grazing is deeply rooted in the nomadic life of the Bedouin and, in recent years, the pressure on ibex habitat has been intensifying because of the reduced mobility of 
Bedouin livestock herders (Hatough-Bouran and Disi, 1991). In addition, a sharp increase in goat and sheep numbers during the past 50 years has been documented by the Ministry of Agriculture (Hatough-Bouran and Disi, 1991). Because the few observed ibexes in DNR appeared restricted to the most inaccessible areas of the reserve, it appears that they are forced to use marginal and less productive habitats to reduce competition with livestock. This corroborates previous findings by Habibi (1994), who maintained that the decline of the Nubian ibex population over its entire range appears to be related mostly to the loss of habitat and to a decreased availability of pasture following an increase in human population and livestock numbers. In addition, on the basis of reported information, it appears that hunting by humans is also believed to represent a potential cause of decline, although its effect on the ibex population in DNR has not yet been assessed.

For the ibex in DNR, prompt conservation efforts need to be made, both for the population itself and for the habitat. These efforts need to be implemented within a broader conservation strategy, which includes planning, control and regulation of human activities. Also, from a conservation perspective, largerscale research is needed to assess the degree of connection (i.e. demographic and genetic exchange) between the population in DNR and others in western Jordan and Israel. For example, Wadi Mujib Wildlife Reserve, which is situated north of DNR and is free of human settlements, is home to an uncensused population of Nubian ibex, whose connection with the DNR population is unknown.

In conclusion, survival of a low-density Nubian ibex population in DNR has been confirmed by our survey. The remnant population appears in need of prompt conservation efforts and, among the potential measures, habitat improvement and protection from livestock grazing appear to be the most immediate priorities. Further research, both at a local and a larger scale, is required to assess the reproductive status and probability of survival of the ibex population.
G. Catullo

Department of Human and Animal Biology Viale dell'Università 32, 00185 Rome, Italy

P. Ciucci

Department of Human and Animal Biology Viale dell'Università 32, 00185 Rome, Italy

A. M. Disi

Department of Biological Sciences Faculty of Science, University of Jordan

Amman, Jordan

L. Boitani

Department of Human and Animal Biology Viale dell'Università 32,00185 Rome, Italy

\section{Acknowledgements}

We acknowledge Adnan Budieri, Edoardo Zandri and the staff members of the RSCN, Jordan, for their continued and valuable support, and Fayes Mashaqbeh, University of Yarmuk, who assisted with fieldwork.

\section{References}

Catullo, G. and Ciucci, P. 1995. Ibex (Capra ibex nubiana). Phase I: November 1994-January 1995, Survey Report. Unpublished report to the Royal Society for Conservation of Nature, Amman, Jordan.

Fox, J., Sinha, S. and Chundawat, R. 1992. Activity patterns and habitat use of ibex in the Himalaya mountains of India. J. Mammal. 73 (3), 527-534.

Groombridge, B. (ed) 1993. 1994 IUCN Red List of Threatened Animals. IUCN, Gland, Switzerland and Cambridge, UK.

Habibi, K. 1994. The Desert Ibex. National commission for Wildlife Conservation and Development, Saudi Arabia and Immel Publishing, London.

Harrison, D.L., Bates, P.J.J. 1991. The Mammals of Arabia, 2nd edn. Harrison Zoological Museum, Sevenoaks.

Hatough-Bouran, A. and Disi, A.M. 1991. History, distribution and conservation of large mammals and their habitats in Jordan. Env. Conserv. 18, 19-33.

RSCN. 1994. Dana Nature Reserve. Baseline Ecological Survey. RSCN Research and Survey Section, Amman, Jordan.

Schaller, G. 1977. Mountain Monarchs: Wild Sheep and Goats of Himalayas. University of Chicago Press, Chicago. 